\title{
The Analysis and Research of the Architecture Landscape Hand Drawn Performance Techniques
}

\author{
Haijiao Xu \\ Jilin business and technology college \\ 346591653@163.com
}

Keywords: The landscape drawing, Performance techniques, The analysis and research

\begin{abstract}
. the architectural landscape hand drawn performance is the most direct method for designers to show emotions and concepts. It is a form of expression, displaying the formation process of the designer's thought. It is simple, fast and direct, and shows the design with thinking, and to be lived, expressing a strong artistic temperament and unique visual觉冲击力。Impacting force.
\end{abstract}

\section{Introduction}

The hand drawn performance and the architectural landscape design have a relationship of dialectical unity. They are the unity of form and thought. Both of them are contained in the designing process. The former Is the catalyst for a design idea to form, promoting the formation process of the latter; while the latter can improve the former, making it practical, accurate and perfect.

This paper elaborates on the basis of the related concepts, exploring and analyzing the pre- , mid- ,and post-production processes of the hand drawn performance.

\section{Conception and function}

Conception. Hand drawn display is a visual communication means, which is that a designer expresses his ideas and concepts of design through hand drawn graphics. It's the carrier of the process of forming and expressing the design ideas, the catalyst for the formation of design ideas. And it's also the unity of the form and the design concept.

Function. First of all, the hand drawn performance promotes the formation of the originality. The performance charts of the landscape architecture design can display the spatial structure of the environment.

They can convey the designer's innovative ideas accurately, and help the designing stuff comprehensively deliberate the space from multiple perspectives.

This technique helps the designing stuff reflect quickly the ideas in their minds, and capture the moments of inspiration.

At the same time, the whole thinking procession of the designer is recorded with extremely strong artistic appealing.

Second, the hand drawn performance helps deliberating and improving the ideas. The freehand sketching is a part of the architects' language. It helps the architects to refine, improve the ideas, and record their inspiration. Freehand sketching can help the teachers and the students better proceed their dialogues. And inspire the students to learn and think in the way of scene demonstration. 
Finally, the hand drawn performance is a bridge to exchange design ideas, through which the designers visually convey the art conception, intentions, and ideas of their design. It's the direct link between party A and party B to communicate and exchange their design ideas. It's the most direct visual language of the engineering technology for designers to express their design ideas.

\section{Characteristics of hand drawn performance}

Artistic quality. Although the hand drawn performance can not be equated with the pure artistic drawing performance, it after all has an inseparable relationship with art.

A beautiful landscape architecture design performance graphic can also be regarded as a very strong visually pleasing art works,

The artistic laws embodied in the drawing are also suitable for the performance of the graph, such as the overall unity, contrast and harmony, order and rhythm, rhythm of change , etc..

The basic problems in drawing, such as relations between sketching and the colors, the relationship between void and reality in the picture, the composition rules etc, also occur in the performance charts.

The space atmosphere, the artistic conception and prospects, the change of color tones expressed in the landscape architecture design charts are also completed by means of drawing skills.

Simple and fast. The designing inspiration of the designers to express their creative thinking can be enriched by a pen and a piece of paper. A few outlines convey intuitively and fast the intentions of the designers' designing, which also can't be replaced by the computer operations.

In the designing process, hand drawn performance chart is the best tool to develop the design thinking, which displays the symbols in the designers' minds vividly on the paper. It's also convenient for the designers to complete their design schemes conceptually.

Originality. Hand drawing is to serve the design, increasing the means of performance for the designers to express their originality.

Come to see some design drawing of the masters in the primary creative stage. Although some of them are fragmental records, made at will, they are also enough to express the designers' design concepts.

Then follow the thorough design. Eventually the creative works of good designs come into being one by one.

The Scientific nature of hand drawing. The space perspectives in the hand drawn performance charts are accurate and more rigorous, which can make the space interfaces and the scales of the landscape facilities strictly accurate. In addition, the drawing material also need to have inherent structure and colors so as to react actually to the changes of the lights and shadows of the building.

\section{The preliminary stage of the hand drawn performance techniques of the architecture landscape}

Adjustment for concepts of the sketch schemes. The architecture landscape scheme design is a creative process which develops from nothing. In the process, the building intentions are blended into the design, and the concrete objects are abstracted. Usually a good scheme in the early stage will be adjusted at least more than five times.

In the process of concepts creation, it's difficult for much information depending on words to be expressed clearly and quickly, and it's also easy to miss some flashes of inspiration. But hand drawing can quickly turn a certain design content into a simple graphic. At the same time, 
experiencing each time of adjustment, many frizzy concepts and graphics in the preliminary stage become clearer, and become full and accurate through constantly adjusting.

While deliberating the concepts of a scheme is an interactive process. Because the precision is emphasized in computer mapping, the fuzzy concepts at the beginning make so strong pressure on the design personnel using computer software that they may lose the interests of design. Take" the four sides of water to the atrium" (When it rains, water all around from the closed surrounding goes down to the atrium.)in the Huizhou architecture as an example. It takes just 30 seconds to display it on paper by hand drawing, while by computer mapping, it will require inputting the detailed sizes and modeling, which consumes a lot time and work.

Communicate design ideas. Communicating time and opportunities between party $\mathrm{A}$ and the designers' party are valuable. During the communication, both sides demand the designers' party to record party $\mathrm{A}^{\prime}$ opinions through quick sketching by hand drawing, and make rapid changes in the drawing.

The effects of tension and imagination expressed by the computer made semi-finished works are far less than by hand drawn performance sketches.

For example, in a practical scenario, the designers ' party proposes to hang up the woodcut Lingnan fruit patterns on the landscape walls, while party A hopes to use the traditional grains to frame the hollow-out scenery-admiring windows. Hand drawn concept sketches can quickly show the situation where the hollow-out scenery-admiring windows have come into being. It solves a lot of problems within a short period of time and accelerates the speed of the overall project of design and construction.

Status records of the surrounding environment. The effects of the surrounding environment on the design subject need to be comprehensively considered in the design of the architecture landscape, including the outer natural factors, the sites' own restrictions, and the social factors and so on. This information has important effects on the design. Because many design companies are far away from the design sites, they use the camera, and video to record, in addition, hand drawing is also a commonly used method to record the complete environmental conditions. It can make many immaterial factors, such information as the history, the traditional cultural folks, etc. collected in the drawings, which makes convenient for the designers to think, and make a deep understanding of the advantages and disadvantages of a good scheme.

\section{The middle stage of the hand drawn performance techniques of the architecture landscape}

Watercolor. Watercolor is a more traditional and higher hand drawn performance form on the post period of the architecture landscape performance. For it depends on water to paint, the color orders will usually be generally distinguished. Watercolor performance needs to use the watercolor paints, water, brushes, wet towel, toilet paper, and even hair dryer. It's relatively complicated. Mastering this kind of performance techniques needs mastering the basic skills and knowledge of drawing. The young designers and the students in the school should initially strengthen their practicing watercolor. Because watercolor effectively improves the students' understanding and perception of the colors and helps them make the color proper light and heavy. Mastering watercolor can also help students get used to using mark pen to make a drawing from light to dark. Depending on different design subjects, it's recommended that, in the sites with larger area, and the broader and more magnificent scenes, designers can make use of the soft transition characteristics of the watercolor to complete the whole picture with the aid of rough brushwork lines, ignoring the small details. As for the small scenes, restrained by the not hard enough tips of the watercolor 
pen , the user can not use his strength, so many beginners can't skillfully control the performance in that case.

Mark pen. Mark pen is also known as the markers. It's a new drawing tool appearing with the process of science and technology in recent years. The color is made harmonic in the pen tube in advance, and comes out evenly through a baize on the tip, it has a single head or a double head. It's more welcome in the hand drawing design performance skills training. Mark pen can be divided into 2 kinds, the oil or the water ones, with the characteristics of drying quickly, no need to use water to make the color harmonic, coloring easily, and drawing fast.

The scope of hand drawn performance objects expressed by Mark pen is very broad. It's suitable both to the small scene and the large-scale scene. Generally speaking, when the construction is taken as the main body to be expressed, especially a large construction, the strokes generally require strengthening, and the trends of the color lines by Mark pen can be seen clearly. As for the entourage of surrounding plants, the strokes should be diffused off in the way of uniform. If it is the rendering of a plant landscaping or the landscape of a small scene, the spatial relationship should be controlled, and comparison between void and the reality should be emphasized as the priority factor. Some color of the sky, some characters can be drawn in order to make the atmosphere of the picture active.

The used paper is related to the mark pen drawing. Drawing paper and copy paper can better reflect the magnitude of the stroke. While the vegetable parchment holds the color, and keeps the traces of the color when it begins to melt. They should be used separately according to different situations.

The pen ink line. Pen ink lines performance here refers to especially the art form of drawing on paper by needle tube pen , water-based pen, and pen. In the post period of the scheme, it's "casual" performance reduces, and its "rational" performance increases. The proportion of using purely the pen and ink lines isn't particularly high in the modern architecture scheme. It usually appears in the repair and reconstruction projects of the traditional Chinese architecture landscape and the design of the new Chinese architecture landscape scheme.

The excellent pen ink line works can reflect the quiet and pleasant atmosphere of the Chinese traditional architecture courtyard. It wins by skills and cleverness. However, restrained by the artistic taste, in the actual projects, many bosses from party A face to this kind of performance blankly.

But that doesn't affects the importance of the pen ink line performance in the landscape architecture discipline. Almost all the color performance need the support from the pen ink lines. We can say that, a piece of good and rich pen ink line manuscript is the foundation of a piece of outstanding works. Simply add some color to several localities, it will make the whole work refreshed.

Colored pencil combined with mark pen. The cooperation between colored pencil and pen applied to the post period of architecture landscape performance design is the intro-industry trends in recent years. Both sides complete each other. The soft colored and colorful pencil makes up the defects of mark pen which has only fixed color. And the large area of stroke made by mark pen also makes up the defects of slow drawing speed made by colored pencil.

For example, in the plane graph of drawing scheme, yellow and green color pencils are used to paint a layer in the plants area as the base color, then similar colors are drawn by mark pen. In this case, the color of mark pen will not be too much bouncing and abrupt, and the lower layer color by the colored pencils will become softer with the aid of the color water from mark pen. 
In the rendering, gray tone of mark pen is used to set a tone for the whole picture. Along traces of mark pen, colored pencil is used to make further extension to several localities. And the picture needn't be completely filled in the process of performance. Some blank left at some localities makes it suitable to perform the sense of space.

\section{The post stage of the hand drawn performance techniques of the architecture landscape}

Make hand drawn performance works into source materials. Hand drawn artistic performance has a natural affinity. So the author here suggests that, during free time more material should be prepared for the post period of software production. Materials can be divided into rendering, plane, elevation, and section, which should be clean and tidy so as to be convenient to be made into cutouts.

In the rendering, the hand drawn materials are mainly used as entourage. According to the experience, at least 5 kinds of materials including the foreground trees, the middle ground and entourage trees, the single tree in the main view, different modeled shrubs, and the combination of ground covers should be prepared, with different attitudes, increasing the sense of sight perspectives for the whole scheme rendering, as well as the background trees whose forms are no need to dig into, and a number of different dynamic characters, adding atmosphere to the rendering.

The material contents for plane, elevation, and section are relatively simple. A variety of tree species in the flat state, big monomer arbors, shrubs, ground covers, and trees connecting the background, with different attitudes, as well as the commonly formative lotus ponds, guardrails, and barrier walls and so on should be prepared. For the rest part, a series of computer actions of selecting, copy, and paste should be used to deal with the lawn, pool, road pavement, architecture, and landscape yard etc. In normal conditions, as long as the hand drawn artistic presentation drawing with rich content is selected as the foundation drawing, the software can be used to complete the corresponding flat or vertical profiles in the presentation drawing.

Stimulate the hand drawn performance with the aid of new types of software. The appearance of the hand drawn plate provides conditions for hand drawing creation through software. It's a product of landmark. It makes use of the pressure pen to draw on the computer, which gets rid of the restriction that the traditional hand drawing needs fixed pen and paper.

Meanwhile, PAINTER produced by COREL for the hand-drawn plate provides various forms of strokes such as crayons, oil paints, colored pencils, and even mark pens etc. PIRANESI is a drawing software specifically designed for SKETCH UP. It can increase the hand drawing effects after the whole model has already had a rudiment, making it have the artistic feeling of hand drawing eventually.Conclusion:

The architecture landscape hand drawn performance techniques can be improved through right ways, with appropriate training. In addition, the drawers need to practice constantly copying, sketching, and making scheme designs. In the practical process, they can make thorough analysis and open their minds. They should also attempt various expressions positively and be able to draw inferences about other cases from one instance so as to produce innovative works with unique connotation, flashing charm of personalities.

\section{References}

[1] Hu Changlong etc. Landscape hand-drawn performance techniques [M] China Machine Press. 2006 
[2] Hong Kong Ke Xun (science and communication) international publishing co., LTD (ed.) . Hand-drawn landscape performance. Huazhong University of Science and Technology Press. 2006

[3] Xia Keliang . Pen architecture sketches and creation [M]. Southeast University Press, 2005

[4] Liu Yu and Ma Zhenlong compiled, Modern environment art representation tutorial [M]. China Planning Press. 2005 